\title{
ON THE CONVECTIVE NATURE OF ROLL WAVES INSTABILITY
}

\author{
C. DI CRISTO AND A. VACCA
}

Received 17 November 2004 and in revised form 25 January 2005

A theoretical analysis of the Saint-Venant one-dimensional flow model is performed in order to define the nature of its instability. Following the Brigg criterion, the investigation is carried out by examining the branch points singularities of dispersion relation in the complex $\omega$ and $k$ planes, where $\omega$ and $k$ are the complex pulsation and wave number of the disturbance, respectively. The nature of the linearly unstable conditions of flow is shown to be of convective type, independently of the Froude number value. Starting from this result a linear spatial stability analysis of the one-dimensional flow model is performed, in terms of time asymptotic response to a pointwise time periodic disturbance. The study reveals an influence of the disturbance frequency on the perturbation spatial growth rate, which constitutes the theoretical foundation of semiempirical criteria commonly employed for predicting roll waves occurrence.

\section{Introduction}

High velocity flows in steep channels may exhibit surface instabilities, perceivable as a train of water waves. As these waves propagate downstream they increase in amplitude and eventually break forming hydraulic bores. The series of waves just described are the so-called "roll waves" and occur usually in man-made channels, such as draining systems and dam spill ways. Generally, roll waves presence is undesirable since they may determine a water overflow from the channel sides and excessive intermittency at the outlet. In studying this phenomenon, two basic issues arise:

(1) identifying the necessary conditions under which roll waves appear;

(2) predicting the roll waves evolution along the channel.

Only the former question will be addressed herein.

From the theoretical point of view, the roll waves generation process has been thoroughly and deeply investigated. In what follows the discussion is restricted to analyses performed with reference to the linearized one-dimensional flow model (Saint-Venant equations) in which the field variables, mean velocity, and flow depth are assumed to be smooth. Under such assumptions roll waves generation process has been fruitfully interpreted in terms of instability of the linearized flow model. 
Many temporal linear stability analyses have been performed $[1,2,6,14]$ in order to individuate the critical values of relevant dimensionless Reynolds and Froude numbers corresponding to the appearance of roll waves. For instance, assuming the channel to be sufficiently wide and expressing the bottom shear stress as a quadratic function of the mean velocity, a critical value of the Froude number, $F_{c}=2$, below which roll waves cannot occur, has been found [6]. Furthermore, all conditions of flow characterized by $F>F_{c}$ are unstable, independently of the perturbation wave-length values. Several experiments (see, e.g., [5]) confirmed the absence of roll waves for $F<F_{c}$, while their presence was not always detected for $F>F_{c}$.

In contrast with temporal analysis and to take into account the developing character of roll waves, spatial linear stability analyses have been performed, starting from the hyperbolic character of the Staint-Venant equations $[9,10,18,19]$. The pointwise perturbation is assumed to propagate downstream with the celerity of the faster shallow water wave, according to the hyperbolic wave theory.

In terms of stability region the results of the temporal analysis are essentially confirmed, despite the strong different space-time evolution of the perturbation.

Whether temporal or spatial technique is more appropriate to investigate the instability of Saint-Venant flow model depends on the nature (convective or absolute) of the instability [8]. An instability is defined as convective if a localized initial small perturbation, growing in time, is swept away from the source only in one direction, whereas the instability is absolute if it spreads in all directions. Only when the instability is convective, spatial analysis may describe fruitfully its evolution.

Even in a linear analysis framework, definition of the instability nature allows to verify the theoretical assumptions embodied in some semiempirical criteria used for defining the minimum channel length below which the roll waves presence is not detected. These criteria, tested and calibrated using many available experimental data, start from the space-time evolution of perturbations resulting from the spatial linear hyperbolic analysis.

Montuori [11, 12], and later Liggett [10], proposed to relate the critical Froude number value with the channel length $L$; for sufficiently small values of $L$ no roll waves appear.

Considering the process as Reynolds independent, in a wide channel the critical Froude number is expressed as a monotone decreasing function of the channel length tending to the limit $F_{c}=2$, for sufficiently large $L$. Similar conclusions have been drawn by Julien and Hartley [9] in studying the roll waves formation process in highly viscous mud flows observable in steep mountain channels. It is worth noting that the above semiempirical criteria share some similarities with the $e^{n}$-method, originally proposed by Van Ingen [17] and by Smith and Gamberoni [15], commonly used to give an engineering prediction of the boundary layers transition location.

In the present paper, the convective nature of the instability is proved by examining the linear impulse response of a given uniform base flow. Following the Briggs criterion [4], an analysis concerning the behavior of the spatial branches associated with the dispersion equation is carried out in the complex $k$ and $\omega$ planes. Furthermore, a spatial linear stability analysis in terms of flow response to a pointwise time periodic perturbation is performed. The analysis naturally accounts for dispersive effects. The strict dependence 
on the source frequency of the perturbation spatial growth rate, whose expression constitutes the theoretical starting point of some criteria commonly employed in hydraulic engineering applications, is finally discussed.

\section{Governing equations}

The system under investigation is an incompressible fluid flowing in a broad rectangular channel, without lateral inflow or outflow and with a nonerodible plane sloping bed. The one-dimensional equations governing the motion are the well-known Saint-Venant equations

$$
\begin{gathered}
\frac{\partial u}{\partial t}+u \frac{\partial u}{\partial x}+g \frac{\partial h}{\partial x}+\frac{\tau_{b}}{\rho h}=g S_{0}, \\
\frac{\partial h}{\partial t}+u \frac{\partial h}{\partial x}+h \frac{\partial u}{\partial x}=0,
\end{gathered}
$$

in which $x$ is the streamwise coordinate, $t$ the time, $g$ the gravity, $\rho$ the water density, $u$ the depth-averaged velocity, $h$ the depth of the water surface, $S_{0}$ the bed slope, and $\tau_{b}$ the bed shear stress. A small perturbation $\left(u^{\prime}, h^{\prime}\right)$ is superposed to a base state uniform flow $\left(u_{0}, h_{0}\right)$. The substitution of the perturbed variables in (2.1) leads, after the linearization, to the following problem:

$$
\begin{gathered}
\frac{\partial u^{\prime}}{\partial t}+u_{0} \frac{\partial u^{\prime}}{\partial x}+g \frac{\partial h^{\prime}}{\partial x}+g S_{0}\left(\frac{\tau_{b}^{\prime}}{\tau_{b 0}}-\frac{h^{\prime}}{h_{0}}\right)=0, \\
\frac{\partial h^{\prime}}{\partial t}+u_{0} \frac{\partial h^{\prime}}{\partial x}+h_{0} \frac{\partial u^{\prime}}{\partial x}=0
\end{gathered}
$$

in which $\tau_{b}=\tau_{b 0}+\tau_{b}^{\prime}$, being $\tau_{b 0}=S_{0} \rho h_{0} g$. Expressing the bed shear stress in terms of the bulk velocity square $\left(\tau_{b}=\rho C_{f} \mathcal{u}^{2}\right)$, and assuming a constant value of the friction coefficient $\left(C_{f}=C_{f 0}\right),(2.2)$ may be rewritten in the following dimensionless form:

$$
\begin{gathered}
\frac{\partial \tilde{u}^{\prime}}{\partial \tilde{t}}+\frac{\partial \tilde{u}^{\prime}}{\partial \tilde{x}}+\frac{1}{F^{2}} \frac{\partial \tilde{h}^{\prime}}{\partial \tilde{x}}+\frac{1}{F^{2}}\left(2 \tilde{u}^{\prime}-\tilde{h}^{\prime}\right)=0, \\
\frac{\partial \tilde{h}^{\prime}}{\partial \tilde{t}}+\frac{\partial \tilde{h}^{\prime}}{\partial \tilde{x}}+\frac{\partial \tilde{u}^{\prime}}{\partial \tilde{x}}=0,
\end{gathered}
$$

in which

$$
\tilde{x}=\frac{x S_{0}}{h_{0}}, \quad \tilde{t}=\frac{t S_{0} u_{0}}{h_{0}}, \quad \tilde{u}^{\prime}=\frac{u^{\prime}}{u_{0}}, \quad \tilde{h}^{\prime}=\frac{h^{\prime}}{h_{0}}
$$

and $F=u_{0} / \sqrt{g h_{0}}$ denotes the unperturbed Froude number.

The first order system (2.3) may be easily reformulated only in terms of depth perturbation $\tilde{h}^{\prime}$ :

$$
\frac{\partial^{2} \tilde{h}^{\prime}}{\partial \tilde{t}^{2}}+2 \frac{\partial^{2} \tilde{h}^{\prime}}{\partial \tilde{t} \partial \tilde{x}}+\left(1-\frac{1}{F^{2}}\right) \frac{\partial^{2} \tilde{h}^{\prime}}{\partial \tilde{x}^{2}}+\frac{1}{F^{2}}\left(3 \frac{\partial \tilde{h}^{\prime}}{\partial \tilde{x}}+2 \frac{\partial \tilde{h}^{\prime}}{\partial \tilde{t}}\right)=0
$$


Following the classical linear stability theory, the perturbation $\tilde{h}^{\prime}$ is decomposed into elementary waves like exp $i(k \tilde{x}-\omega \tilde{t})$, with $k=k_{r}+k_{i}$ the dimensionless complex wave number and $\omega=\omega_{r}+i \omega_{i}$ the dimensionless complex pulsation. The substitution of such a perturbation into (2.5) leads to the quadratic dispersion equation

$$
D(k, \omega, F)=F^{2} i(\omega-k)^{2}-k^{2} i-2 \omega+3 k=0 .
$$

In a temporal stability analysis, the wave number $k$ is assumed to be real and unstable conditions are related to the existence of complex frequencies $\omega$, roots of (2.6), with negative imaginary part. On the other hand, in a spatial stability analysis the pulsation $\omega$ is considered real and the instability is related to the occurrence of complex wave numbers $k$, roots of (2.6), with positive imaginary part.

In order to ascertain which of the two techniques is the most appropriate to describe an unstable process, a study on the response of the flow model to a wave packet is needed. The analysis of asymptotic temporal behavior of the solution allows to classify the instability nature as convective or absolute. In the convective instability an initial disturbance, localized in space, is swept away from the source. By contrast, instability is absolute if a preferential direction of propagation does not exist. In such an instance the disturbance spreads both in downstream and upstream directions contaminating the whole domain as time tends to infinity. A spatial analysis leads to a well-posed problem only when the instability is of convective type. Contributions to the theoretical foundations underlying these notions have been brought out in studies concerning plasma [3] and hydrodynamic stability (see the review of Huerre and Monkewitz [8]). In a morphodynamic context, the nature of bar formation process has been recently investigated by Federici and Seminara [7].

\section{Convective nature of instability}

The nature of instability of the linearized Saint-Venant flow model may be ascertained by studying the impulsive response of (2.5). The usual approach leads to investigating the asymptotic time behavior of the solution (Green's function) of the following initial value problem:

$$
\frac{\partial^{2} G}{\partial \tilde{t}^{2}}+2 \frac{\partial^{2} G}{\partial \tilde{t} \partial \tilde{x}}+\left(1-\frac{1}{F^{2}}\right) \frac{\partial^{2} G}{\partial \tilde{x}^{2}}+\frac{1}{F^{2}}\left(3 \frac{\partial G}{\partial \tilde{x}}+2 \frac{\partial G}{\partial \tilde{t}}\right)=\delta(\tilde{x}) \delta(\tilde{t}),
$$

where $\delta$ is the Dirac delta function. The spatial domain is assumed to be unbounded and homogeneous initial conditions are prescribed. Following Huerre and Monkewitz [8], the base flow is classified as linearly unstable if there exists a ray $\tilde{x} / \tilde{t}=$ cost along which

$$
\lim _{\tilde{t} \rightarrow \infty} G(\tilde{x}, \tilde{t})=\infty
$$

A linear unstable condition of flow is convective if, along the ray $\tilde{x} / \tilde{t}=0$,

$$
\lim _{\tilde{t} \rightarrow \infty} G(\tilde{x}, \tilde{t})=0,
$$


whereas it is absolutely unstable when, along the ray $\tilde{x} / \tilde{t}=0$,

$$
\lim _{\tilde{t} \rightarrow \infty} G(\tilde{x}, \tilde{t})=\infty
$$

The asymptotic behavior of the Green function (3.2)-(3.4) may be analyzed representing $G(\tilde{x}, \tilde{t})$ as double Fourier integral in the complex $\omega$ and $k$ planes

$$
G(\tilde{x}, \tilde{t})=\frac{1}{(2 \pi)^{2}} \int_{L} \int_{C} e^{i(k \tilde{x}-\omega \tilde{t})} \frac{d k d \omega}{D(k, \omega, F)}
$$

in which the contours $L$ and $C$ are placed in the region of absolute convergence of $G(\tilde{x}, \tilde{t})$ and $D(k, \omega, F)$ is the dispersion operator given by (2.6). The application of the steepest descend method to (3.5) allows to analyze the asymptotic conditions (3.2)-(3.4) only through the study of both temporal $\omega(k)$ and spatial $k(\omega)$ branches of the dispersion relation $D(k, \omega, F)$ in the complex $\omega$ and $k$ planes $[4,8]$.

It may be shown [8] that condition (3.2) is not fulfilled, that is, linearly stable conditions of flow occur, if $\omega_{i}\left(k_{r}\right)<0$ for all $k=k_{r}$ and for all temporal branches $\omega\left(k_{r}\right)$.

Therefore, the presence of linearly unstable conditions of flow will be detected if the maximum value $\omega_{i}^{\max }$ of $\omega_{i}\left(k_{r}\right)$ is positive definite.

The simple structure of the dispersion equation (2.6) allows to deduce in an analytic form the temporal branches $\omega(k)$,

$$
\omega=k-\frac{i}{F^{2}} \pm \sqrt{\left(k-\frac{i}{F^{2}}\right)^{2}-k^{2}\left(1-\frac{1}{F^{2}}\right)+3 i \frac{k}{F^{2}}} .
$$

Setting $k=k_{r}$ in the right-hand side of (3.6), it follows that, according to previous results [6], linearly unstable conditions of flow are permitted only when the Froude number exceeds the limit value $F_{c}=2$.

Figure 3.1, in which both temporal branches $\omega\left(k_{r}\right)$ are depicted in the complex $\omega$ plane for three different Froude number values, namely, $F=0.5, F=1.5, F=2.5$, clearly shows the instability occurrence only in the $F=2.5$ case.

In order to ascertain the nature of unstable conditions of flow, occurring when $F>F_{c}$, a careful study of the spatial branches is required. Briggs [4] proved that the absolute character of the instability may be defined looking for the complex frequencies $\omega^{0}$ for which two (or more) spatial branches $k(\omega)$ merge (pinch-type singularities). The positive definiteness of $\omega_{i}^{0}$ is the necessary condition for the absolute character of the instability. The sufficient condition comes from the analysis, for large enough $\omega$ values, that is, $\omega_{i}>$ $\omega_{i}^{\max }$, of the spatial branches $k(\omega)$ that coalesce for $\omega=\omega^{0}$. The instability will be of absolute type if for $\omega_{i}>\omega_{i}^{\max }$ at least two of such branches are placed on opposite sides of the real $k$-axis. The study of the branches location, in condition of spatial evanescence $\left(\omega_{i}>\omega_{i}^{\max }\right)$, ensures that the instability, corresponding to the pinch, will appear as two waves, propagating in opposite directions, without any discontinuity at the origin of the spatial coordinates. The time growth of these waves, that behave as only one wave (normal mode), will lead to the contamination of the whole domain as time tends to infinity. 


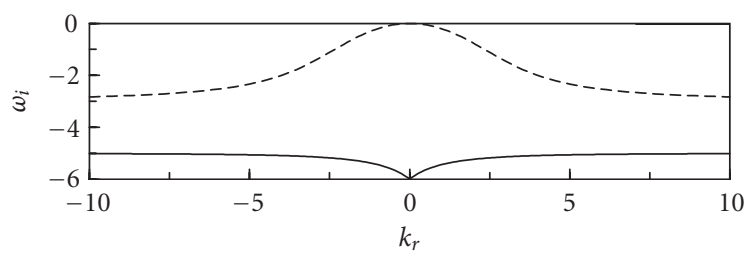

(a)

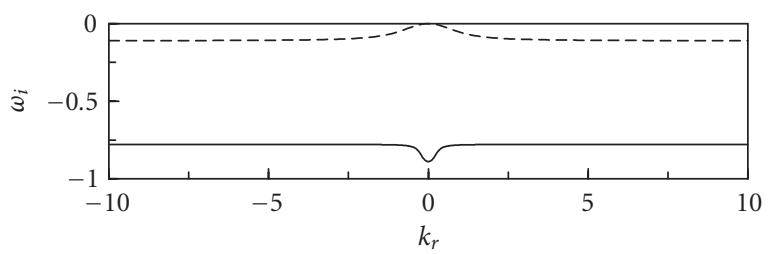

(b)

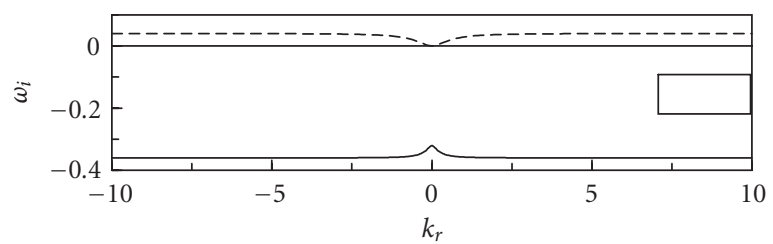

(c)

Figure 3.1. Imaginary part of temporal branches, where in (a) $F=0.5$, in (b) $F=1.5$, and in (c) $F=2.5$.

Since the spatial branches merging points correspond to the saddle points of the temporal branches $\omega(k)$ [8], the necessary condition of the Briggs criterion may be analyzed alternatively looking for the complex wave number $k^{0}$ such that $d \omega / d k=0$, that is, $\omega^{0}=\omega\left(k^{0}\right)$. The last technique is the most useful when many spatial branches are present.

The above procedures may be applied only when pinch-type singularities occur at finite values of $k^{0}$. If such an instance does not occur, the singularities will be defined as essential and the Brigg criterion cannot be straightforwardly applied. In this case, a direct numerical evaluation of $G(\tilde{x}, \tilde{t})$ is needed [13].

In the Saint-Venant flow model (2.5) only two spatial branches exist,

$$
k=\frac{2 F^{2} \omega+3 i \pm \sqrt{4 F^{2} \omega(\omega+i)-9+8 i \omega}}{2\left(F^{2}-1\right)},
$$




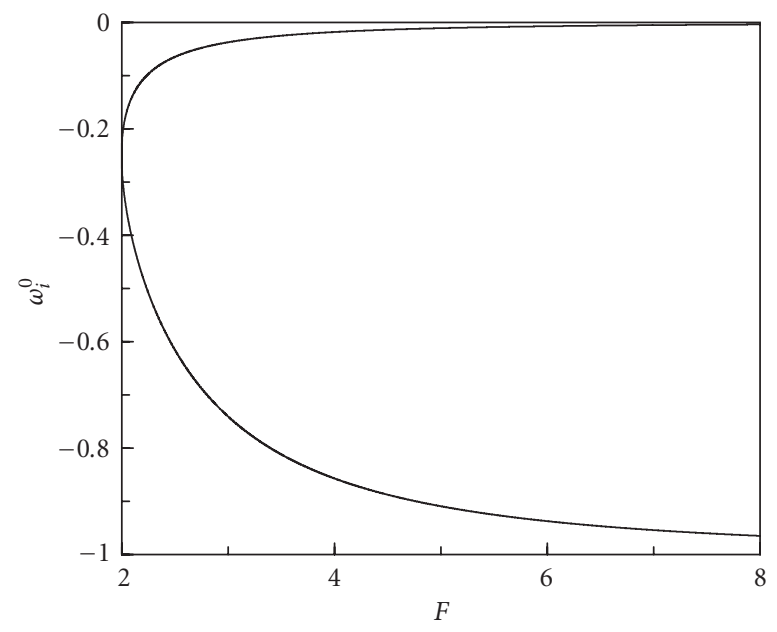

Figure 3.2. Imaginary part of finite pinch-type singularities versus Froude number.

whose merging points are given by

$$
\omega^{0}=\frac{-i\left(F^{2}-2\right) \pm i \sqrt{\left(F^{2}-4\right)\left(F^{2}-1\right)}}{2 F^{2}} .
$$

The inspection of (3.8) and of (3.7) with $\omega=\omega^{0}$ allows to conclude that the instability of the Saint-Venant flow model has a convective nature, since only finite pinch-type singularities characterized by negative imaginary part exist, for $F>2$. Figure 3.2 gives a graphical evidence of the negative definiteness of the $\omega_{i}^{0}(F)$ function.

\section{Response to a pointwise time-periodic disturbance}

The absence of absolute instabilities suggests to analyze roll waves generation process by linear spatial analysis. In this context we investigate, considering the dispersive effects, the time-asymptotic response of the flow model to a pointwise external source oscillating with a prescribed frequency.

Let $\omega_{e}=2 \pi f_{e} / S_{0}$ be the dimensionless pulsation of the oscillation, $f_{e}=h_{0} /\left(u_{0} T_{e}\right)$ being the source frequency, with $T_{e}$ the dimensional period of oscillation. Assuming the flow domain to be unbounded, the mathematical model reads

$$
\frac{\partial^{2} R}{\partial \tilde{t}^{2}}+2 \frac{\partial^{2} R}{\partial \tilde{t} \partial \tilde{x}}+\left(1-\frac{1}{F^{2}}\right) \frac{\partial^{2} R}{\partial \tilde{x}^{2}}+\frac{1}{F^{2}}\left(3 \frac{\partial R}{\partial \tilde{x}}+2 \frac{\partial R}{\partial \tilde{t}}\right)=\delta(\tilde{x}) e^{-i \omega_{e} \tilde{t}}
$$

As in the pulse case, the solution of problem (4.1) may be described as double Fourier integral in the complex $\omega$ and $k$ planes

$$
R(\tilde{x}, \tilde{t})=\frac{1}{(2 \pi)^{2}} \int_{L} \int_{C} e^{i(k \tilde{x}-\omega \tilde{t})} \frac{d k d \omega}{\left(\omega-\omega_{e}\right) D(k, \omega, F)} .
$$


In unstable condition of flow, $F>F_{c}$, the time-asymptotic behavior of (4.2) may be written in terms of two waves traveling both in the downstream direction [3],

$$
R(\tilde{x}, \tilde{t})=i \sum_{l=1,2} \frac{U(\tilde{x})}{(\partial D / \partial k)_{k=k^{l}}} e^{-i\left[\omega_{e} \tilde{t}-k^{l}\left(\omega_{e}\right) \tilde{x}\right]},
$$

where $U(\tilde{x})$ is the unit-step function, $k^{l}\left(\omega_{e}\right) l=1,2$, are the two spatial branches, obtained setting $\omega=\omega_{e}$ in $(3.7)$,

$$
k^{l}\left(\omega_{e}\right)=\frac{2 F^{2} \omega_{e}+3 i \pm \sqrt{4 F^{2} \omega_{e}\left(\omega_{e}+i\right)-9+8 i \omega_{e}}}{2\left(F^{2}-1\right)}
$$

and finally

$$
\left(\frac{\partial D}{\partial k}\right)_{k=k^{l}}=2 i F^{2}\left(\omega_{e}-k^{l}\left(\omega_{e}\right)\right)+2 i k^{l}\left(\omega_{e}\right)-3 .
$$

Independently of the $\omega_{e}$ value, the inspection of (4.4) leads to recognize the existence of only one spatially excited wave $\left(k_{i}\left(\omega_{e}\right)<0\right)$; the other one is spatial evanescent. A graphical evidence of such findings is given in Figure 4.1, in which the imaginary part of the two spatial branches $k^{l}\left(\omega_{e}\right)$, for $F=2.5, F=5.0$, and $F=7.5$, is represented. The analysis of the propagation phase speed $c=\omega_{e} / k_{r}\left(\omega_{e}\right)$ suggests that the excited wave is the primary one (fast), while the evanescent one is the secondary one (slow). In Figure 4.2 the propagation phase speed of both waves versus $\omega_{e}$ is depicted, for $F=2.5, F=5.0$, and $F=7.5$. The diagram reveals that the $c$ value of both waves is not constant and strongly depends on the value of the source pulsation. Similar results have been derived by Supino [16] in studying the evolution of small waves in a hydraulic channel, starting from the linearized Saint-Venant flow model.

We focus the attention on the primary unstable wave. Equation (4.4) indicates that, for a constant Froude number value, the imaginary part of the wave number, that is, the spatial growth rate, is a bounded monotone function of $\omega_{e}$ or, equivalently, of the source frequency $f_{e}$, for a fixed value of the bed channel slope. Independently of the Froude number, very small values of the frequency lead to a vanishing spatial growth rate. On the other hand, the limit process for very high frequencies $\left(f_{e} \rightarrow \infty\right)$ furnishes the minimum asymptotic value of the $k_{i}$, that is, maximum spatial grow rate, that reads

$$
k_{i}^{\min }(F)=\frac{2-F}{2 F(F+1)} .
$$

The propagation phase speed shows a monotone dependence upon frequency $f_{e}$. Its maximum $c^{\max }=1.5$ occurs at $f_{e}=0$, independently of the Froude number, and the asymptotic value $c_{\infty}=1+1 / F$ represents its minimum.

Spatial growth rates, formally equivalent to the asymptotic value (4.6), have been deduced from previous linear analyses which do not consider the dispersive effects $[10,18$, 19]. Moreover, these growth rate expressions have constituted the theoretical justification to semiempirical criteria, for predicting the presence of the roll waves $[9,11]$. 


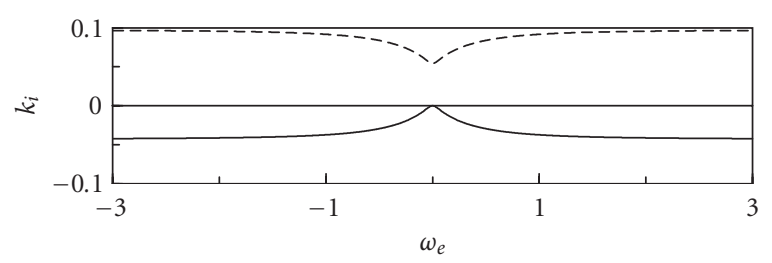

(a)

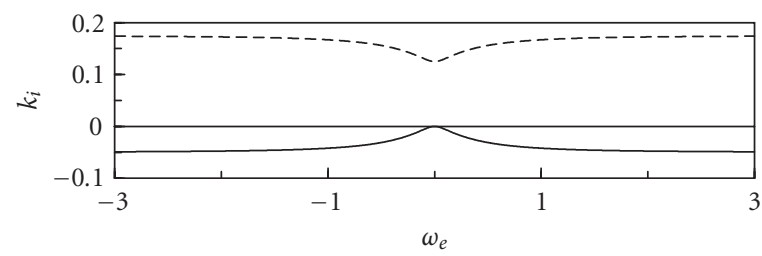

(b)

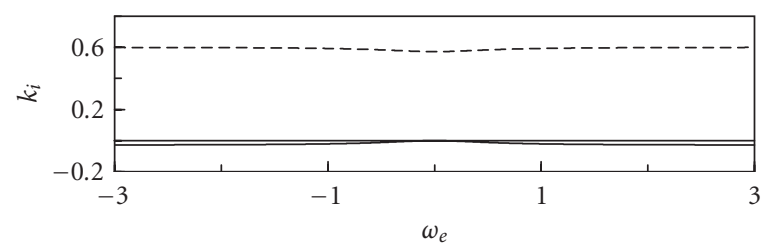

(c)

Figure 4.1. Imaginary part of the wave number. The solid line represents unstable wave and the dashed line represents evanescent wave. (a) $F=7.5$, (b) $F=5$, and (c) $F=2.5$.

In particular, Montuori $[11,12]$ related the formation of the roll waves not only to the Froude number but also to the channel length $L$. Starting from the results of the spatial linear analysis of Vedernikov $[18,19]$, the author introduced a functional dependence of the critical Froude number value upon the nondimensional channel length $L S_{0} / h_{0}$, that, as far as wide rectangular channels are concerned, may be rewritten as follows:

$$
\frac{L S_{0}}{h_{0}}=\frac{\ln (\epsilon)}{k_{i}^{\min }(F)} .
$$

The amplification factor $\epsilon=10^{-4}$ appearing in (4.7) has been calibrated starting from numerous field data collected in Europe and in Russia. The curve defined by (4.7) individuates two disjoint regions, in the $L S_{0} / h_{0}-F$ plane. Roll waves formation is inhibited in flow conditions characterized by $\left(L S_{0} / h_{0}, F\right)$ pairs laying below the curve. 


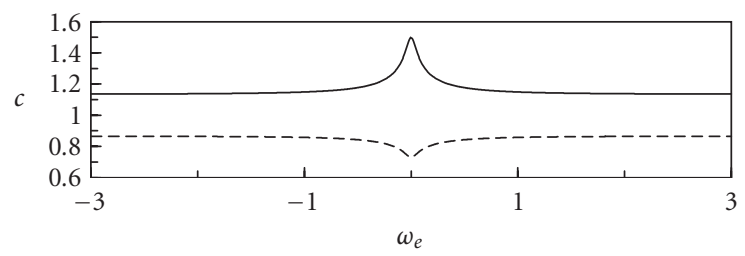

(a)

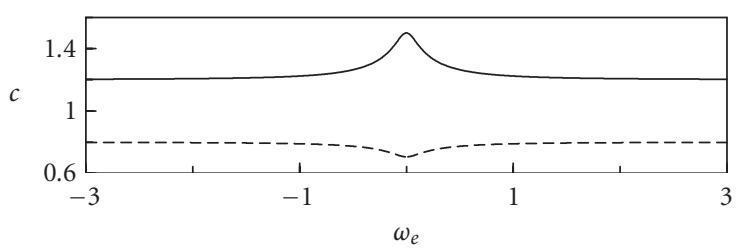

(b)

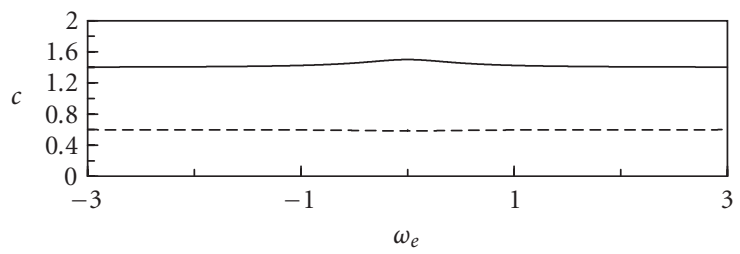

(c)

Figure 4.2. Phase speed. The solid line represents growing wave and the dashed line represents the evanescent wave. (a) $F=7.5$, (b) $F=5$, and (c) $F=2.5$.

Figures 4.3 and 4.4 depict the deviation of the calculated spatial growth rate from its asymptotic value $\left(\alpha=k_{i} / k_{i}^{\min }\right)$ versus the frequency $f_{e}$, for two different channel bed slope values $\left(S_{0}=0.01\right.$ and $\left.S_{0}=0.30\right)$. In each figure, three different values of the Froude number $(F=2.5, F=5$, and $F=7.5)$ have been considered.

In the $S_{0}=0.01$ case (Figure 4.3$)$ only for very low frequency values $\left(T_{e}>500 h_{0} / u_{0}\right)$ substantial deviations from the asymptotic value may be detected. In contrast, for higher bed channel slope value (Figure 4.4) considerable differences appear in a broader frequency range. Independently of the bed channel slope, the deviations reduce when the Froude number is increased, even though the Froude number effect appears to be less significant.

From the above results it follows that the spatial evolution of the perturbation, according to the dispersion effects in a linear approximation context, is governed by three dimensionless parameters $x S_{0} / h_{0}, F, f_{e} / S_{0}$. The conventional approaches employing only 


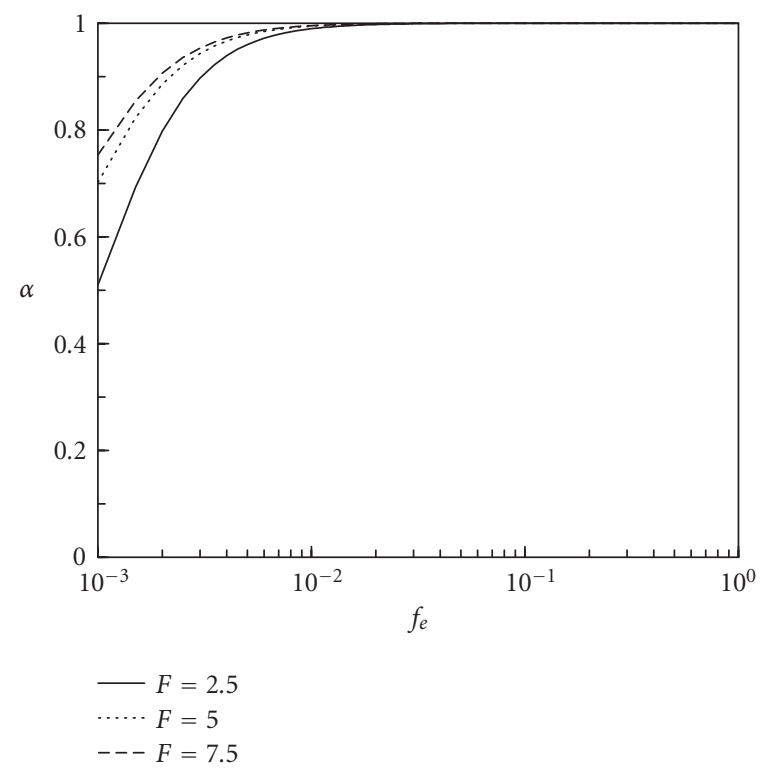

Figure 4.3. Spatial growth rate $\alpha=k_{i} / k_{i}^{\text {min }}$ versus the dimensionless frequency $f_{e}\left(S_{0}=0.01\right)$.

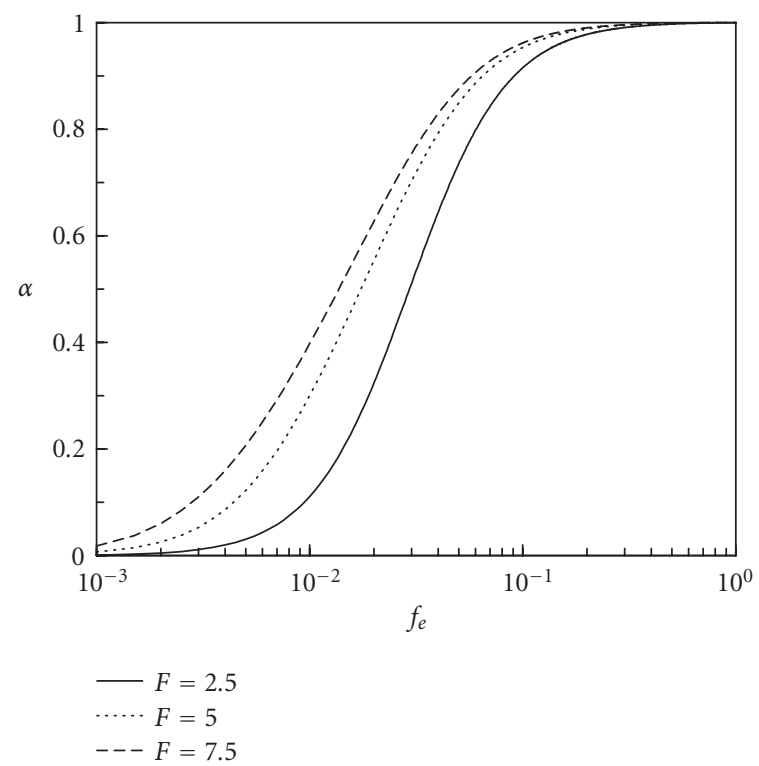

Figure 4.4. Spatial growth rate $\alpha=k_{i} / k_{i}^{\min }$ versus the dimensionless frequency $f_{e}\left(S_{0}=0.3\right)$.

two parameters $x S_{0} / h_{0}$ and $F$ (see, e.g., [20] and discussions) therefore require an additional constraint on the minimum value of the physical disturbance frequency. We wish to stress that such a lower bound may be particularly severe in the presence of channels 
with huge bed slope (see Figure 4.4). Furthermore, neglecting the frequency effects leads to overestimating the spatial growth of the disturbance. This final remark provides a theoretical justification for the experimentally ascertained underestimation of the minimum channel length above which (4.7) predicts the presence of roll waves [11]. As a matter of fact, Figure 4.4 clearly shows that for large bed slopes the amplification factor is considerably reduced compared to its asymptotic value, in large range of dimensionless frequencies $f_{e}$. Therefore, (4.7) would correctly account for the above-quoted experimental evidences, provided $k_{i}^{\text {min }}$ is replaced by the correct spatial growth rate of the unstable wave given in (4.4) and the frequency of the disturbance is not excessively large. Which is the appropriate disturbance frequency value is still, as far as the authors are aware, an open question that requires properly designed experiments or direct numerical simulations.

\section{Conclusions}

In the present paper, the convective instability property of the one-dimensional linearized flow model has been demonstrated. The Briggs criterion, concerning the analysis of the branch point singularities of the dispersion relation, has been applied. Starting from the ascertained convective character of the instability a spatial linear stability analysis has been carried out. Results showed that, in unstable condition of flow, a pointwise temporal oscillating perturbation generates two waves, both propagating in the downstream direction. The primary is spatially growing, the secondary is spatially evanescent. The spatial growth rate of the unstable wave strongly depends on the frequency of the disturbance, particularly as far as high bed slope channels are concerned. It follows that the description of the spatial evolution of the perturbation only through the Froude number and the nondimensional distance, commonly assumed in hydraulic engineering criteria, may lead to overestimating the spatial amplification of the perturbation, depending on the temporal frequency of the disturbance and on the bed slope of the channel.

\section{References}

[1] J. E. Berlamont, Roll-waves in inclined rectangular open channels, Intenational Symposium on Unsteady Flow in Open Channels, vol. A2, BHRA Fluid Engineering, Newcastle, 1976, pp. 13-26.

[2] J. E. Berlamont and N. Vanderstappen, Unstable turbulent flow in open channels, J. Hydr. Div., ASCE 110 (1981), 427-449.

[3] A. Bers, Linear waves instabilities, Physique des Plasmas (C. De Witt and J. Peyraund, eds.), Gordon \& Breach Publishers, New York, 1975, pp. 117-215.

[4] R. J. Briggs, Electon-Steam Interaction with Plasmas, MIT Press, Massachusetts, 1964.

[5] R. R. Brock, Development of roll-waves trains in open channels, J. Hydr. Div., ASCE 95 (1969), 1401-1427.

[6] Periodic permanent roll waves, J. Hydr. Div., ASCE 96 (1970), 2565-2580.

[7] B. Federici and G. Seminara, On the convective nature of bar instability, J. Fluid Mech. 487 (2003), 125-145.

[8] P. Huerre and P. A. Monkewitz, Local and global instabilities in spatially developing flows, Annual Review of Fluid Mechanics, vol. 22, Annual Reviews, California, 1990, pp. 473-537.

[9] P. Y. Julien and D. M. Hartley, Formation of roll waves in laminar sheet flow, J. Hydr. Res. 24 (1986), no. 1, 5-17. 
[10] J. A. Liggett, Stability, Unsteady Flow in Open Channels (K. Mahmood and V. Yevjevich, eds.), Water Resources Publications, Colorado, 1975.

[11] C. Montuori, La formazione spontanea dei treni d'onde su canali a pendenza molto forte, l'Energia Elettrica 2 (1961), 127-141 (Italian).

[12] Discussion: Stability aspect of flow in open channels, J. Hydr. Div., ASCE 89 (1963), 264-273.

[13] D. Park and P. Huerre, On the convective nature of the Goertler instability, Bull. Amer. Math. Soc. 33 (1988), 2552.

[14] V. M. Ponce and D. B. Simon, Shallow water propagation in open channel flow, J. Hydr. Div., ASCE 103 (1977), 1461-1476.

[15] A. M. O. Smith and N. Gamberoni, Transition pressure gradient and stability theory, Rept. ES 26388, Douglas Aircraft company, California, 1956.

[16] G. Supino, Sopra le onde di traslazione nei canali, Rendiconti Lincei XXIX (1960), no. 5-6, 543-552 (Italian).

[17] J. L. Van Ingen, A suggested semi-empirical method for the calculation of the boundary layer transition, Rept. UTH-74, University of Technology, Department of Aerospace Engineering, Delft, 1956.

[18] V. V. Vedernikov, Conditions at the front of a translation wave disturbing a steady motion of a real fluid, C. R. (Doklady) Acad. Sci. URSS (N.S.) 48 (1945), 239-242.

[19] Characteristics feature of a liquid flow in open channel, C. R. (Doklady) Acad. Sci. URSS (N.S.) 52 (1946), 207-210.

[20] B. Zanuttigh and A. Lamberti, Roll waves simulation using shallow water equations and weight flux method, J. Hydr. Res. 40 (2003), no. 5, 610-622.

C. Di Cristo: Dipartimento di Ingegneria Idraulica ed Ambientale, Universitá di Napoli Federico II, Via Claudio 21, 80125 Napoli, Italy

E-mail address: dicristo@unina.it

A. Vacca: Dipartimento di Ingegneria Civile, Seconda Universitá di Napoli, Via Roma 29, 81031 Aversa (Ce), Italy

E-mail address: vacca@unina.it 


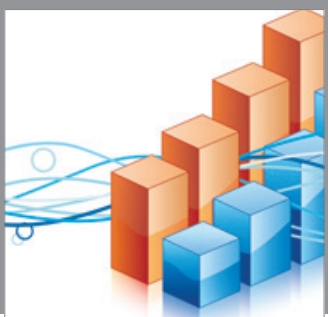

Advances in

Operations Research

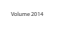

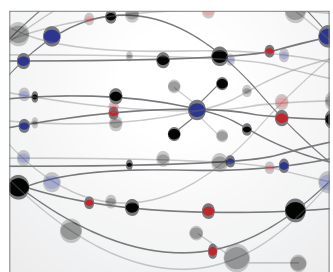

\section{The Scientific} World Journal
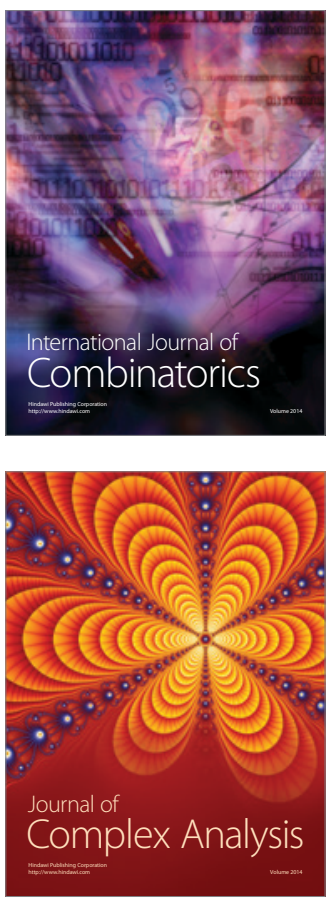

International Journal of

Mathematics and

Mathematical

Sciences
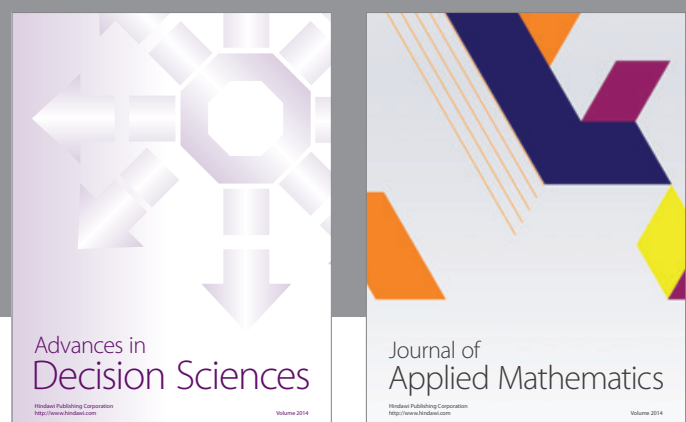

Journal of

Applied Mathematics
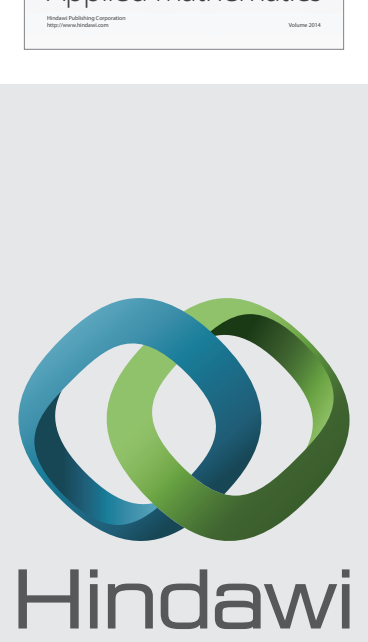

Submit your manuscripts at http://www.hindawi.com
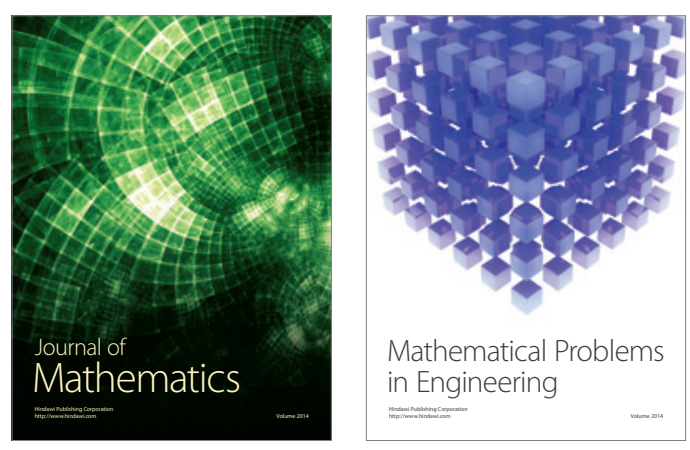

Mathematical Problems in Engineering
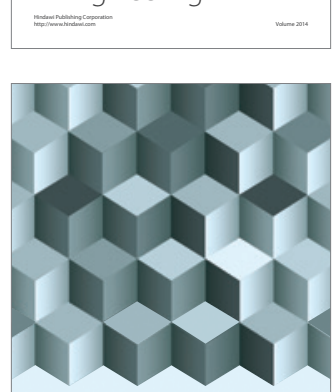

Journal of

Function Spaces
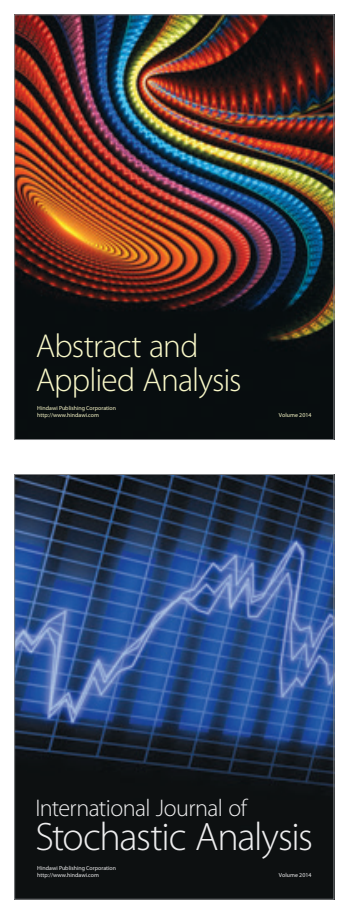

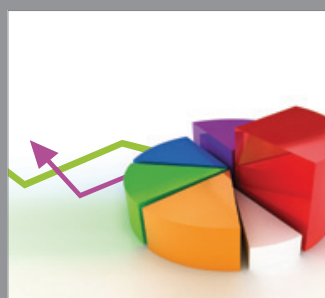

ournal of

Probability and Statistics

Promensencen
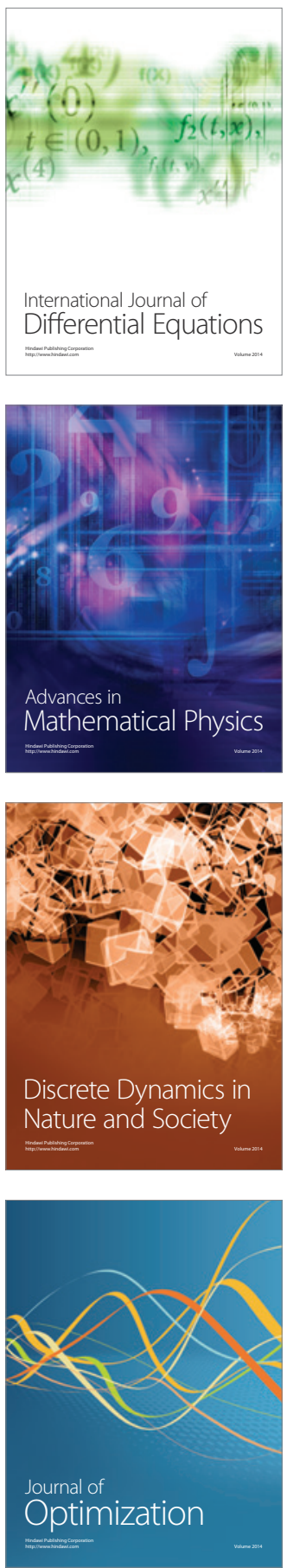